\title{
Family and friends as caregivers in the Neonatal Intensive Care Unit
}

\section{J.E. Dullaert, J.M. Wielenga}

Neonatal Intensive Care Unit, Emma Children's Hospital, Amsterdam university medical centers, location AMC, The Netherlands, j.e.dullaert@amc.nl

\section{Background and aims}

Current NICU care involves extended engagement of family and friends (informal caregivers), as part of the Family Integrated Care philosophy. To make sure that no unwanted choices between the supportive care possibilities are offered to the newborn and the parents, a guideline is necessary. A guideline guides the professional in what can be offered to the parents. But it also gives the parents the opportunity to shape the care of their child as they want and need.

\section{Methods}

Based on the literature topics, to be part of a guideline, were selected. Next focus groups of nurses $(\mathrm{N}=20)$, medical staff $(\mathrm{N}=8)$ and a social worker $(\mathrm{N}=1)$ were initiated to decide on the content of these topics. After every focus group the concept-guideline was updated based on the conclusions of the participants until an approved guideline derived.

\section{Results}

Topics for the guideline were: the criteria to start with informal caregivers; risk and risk reduction (infection prevention, excess stimuli of the infant, excessive burden of care for parents and informal care givers); information for professionals, parents and informal care givers; procedures to be followed. After four focus groups consensus on the content of the guideline was reached. Topics giving the most difficulties where describing the responsibilities of the different parties and identification of parents and the informal care givers.

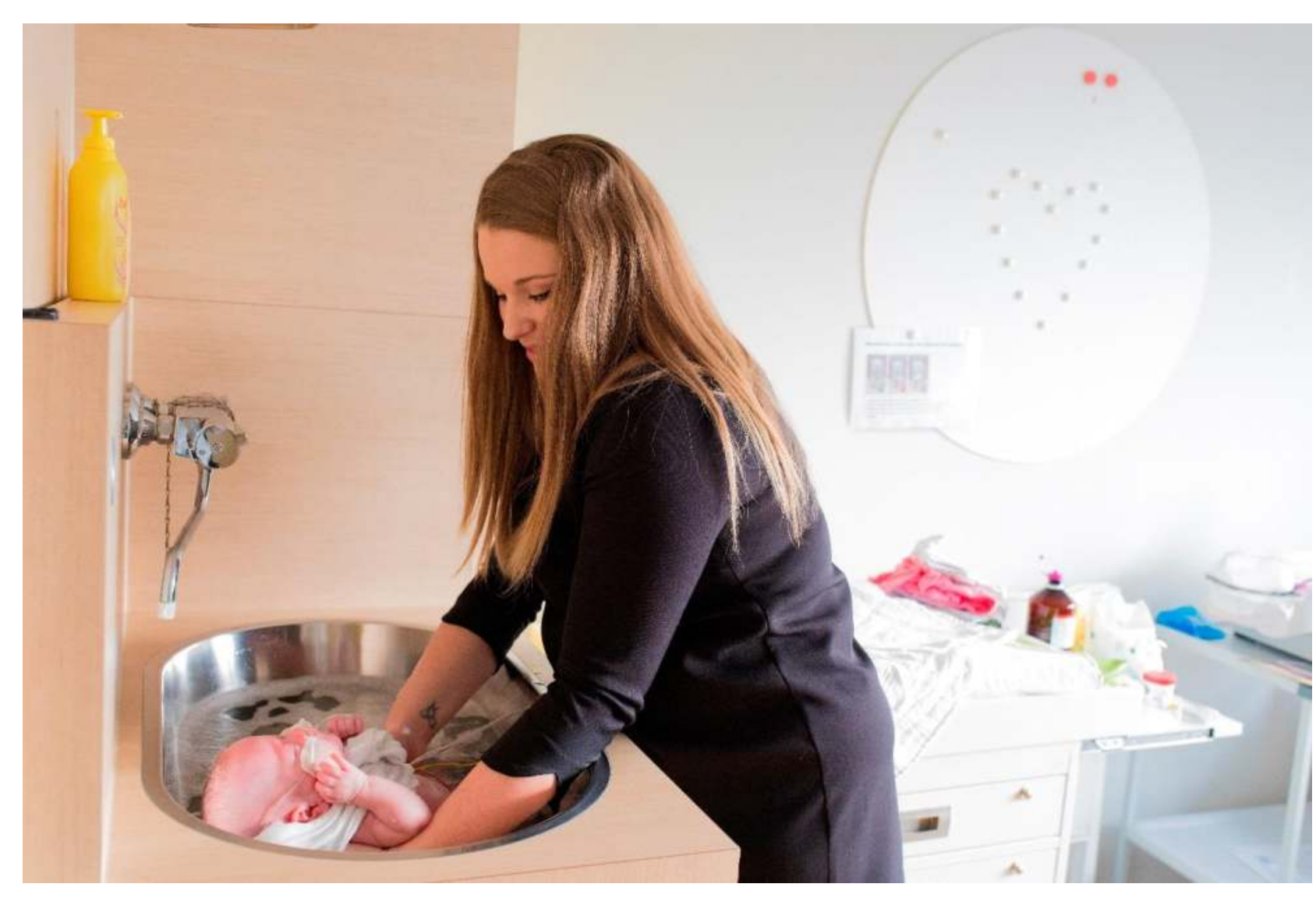

Photographer: Nienke Elenbaas

\section{Conclusions}

A guideline was formulated: nurses, medical staff and social work feel confident to start with the implementation of family and friends as caregivers. 